\title{
INTEGRAL Observations of Gravitational-Wave Counterparts \& Future Perspectives: Searching for GBM Un-Triggered SGRB with PICsIT
}

James Rodi*

INAF, IAPS, Via Fosso del Cavaliere 100, 00133-Rome, Italy

E-mail: james.rodi@iaps.inaf.it

\section{A. Bazzano}

INAF, IAPS, Via Fosso del Cavaliere 100, 00133-Rome, Italy

E-mail: angela.bazzano@iaps.inaf.it

L. Natalucci

INAF, IAPS, Via Fosso del Cavaliere 100, 00133-Rome, Italy

E-mail: lorenzo.natalucci@iaps.inaf.it

P. Ubertini

INAF, IAPS, Via Fosso del Cavaliere 100, 00133-Rome, Italy

E-mail: pietro.ubertini@iaps.inaf.it

\section{S. Mereghetti}

INAF, IASF-Milano, via E.Bassini 15, I-20133 Milano, Italy

E-mail: sandrodiasf-milano.inaf.it

\section{E. Bozzo}

ISDC, University of Geneva, chemin d'Ecogia, 16 CH-1290 Versoix, Switzerland E-mail: Enrico.Bozzo@unige.ch

\section{Ferrigno}

ISDC, University of Geneva, chemin d'Ecogia, 16 CH-1290 Versoix, Switzerland E-mail: Carlo.Ferrigno@unige.ch

\section{Savchenko}

ISDC, University of Geneva, chemin d'Ecogia, 16 CH-1290 Versoix, Switzerland E-mail: Volodymyr.Savchenko@unige.ch

\section{T. J.-L. Courvoisier}

ISDC, University of Geneva, chemin d'Ecogia, 16 CH-1290 Versoix, Switzerland

E-mail: Thierry. Courvoisierdunige.ch

\section{E. Kuulkers}

ESA/ESTEC, Keplerlaan 1, 2201 AZ Noordwijk, The Netherlands

E-mail: erik.kuulkersesciops.esa.int

\section{S. Brandt}

DTU, Building 327, DK-2800 Kongens, Lyngby, Denmark

E-mail: sbespacecenter.dk 


\section{J. Chenevez}

DTU, Building 327, DK-2800 Kongens, Lyngby, Denmark

E-mail: jeromeespace.dtu.dk

\section{R. Diehl}

Max Planck Institute for Astrophysics, Garching, Germany

E-mail: rod@mpe.mpg.de

\section{A. von Kienlin}

Max Planck Institute for Astrophysics, Garching, Germany

E-mail: azk@mpe.mpg.de

\section{Hanlon}

University College Dublin, Belfield, Dublin 4, Ireland

E-mail: lorraine.hanloneucd.ie

\section{A. Martin-Carrillo}

University College Dublin, Belfield, Dublin 4, Ireland

E-mail: Antonio.Martin-Carrillo@ucd.ie

\section{E. Jourdain}

Université de Toulouse; UPS-OMP; IRAP; Toulouse, France; CNRS; IRAP; 9 Av. Colonel Roche, BP 44346, F-31028 Toulouse cedex 4, France

E-mail: elisabeth. jourdain@irap.omp.eu

\section{J.-P. Roques}

Université de Toulouse; UPS-OMP; IRAP; Toulouse, France; CNRS; IRAP; 9 Av. Colonel Roche, BP 44346, F-31028 Toulouse cedex 4, France

E-mail: roquesecesr.fr

\section{P. Laurent}

APC, CNRS/IN2P3, CEA/Irfu, Observatoire de Paris Sorbonne Paris Cite, rue Alice Domont et Leonie Duquet, 75205 Paris Cedex 13, France. and DSM/IRFU/SAp, CEA Saclay, 91191

Gif-sur-Yvette Cedex, France

E-mail: philippe.laurentecea.fr

\section{F. Lebrun}

APC, CNRS/IN2P3, CEA/Irfu, Observatoire de Paris Sorbonne Paris Cite, rue Alice Domont et Leonie Duquet, 75205 Paris Cedex 13, France. and DSM/IRFU/SAp, CEA Saclay, 91191

Gif-sur-Yvette Cedex, France

E-mail: flebruneapc.in2p3.fr

\section{A. Lutovinov}

Space Research Institute, Profsoyuznaya 84/32, 117997 Moscow, Russia

E-mail: aaleiki.rssi.ru

\section{R. Sunyaev}

Space Research Institute, Profsoyuznaya 84/32, 117997 Moscow, Russia

E-mail: sunyaev@iki.rssi.ru 
The X-ray/gamma-ray mission INTEGRAL detected the short GRB170817A and demonstrated its association to a gravitational wave trigger, GW170817. This marks the first time a binary neutron star merger was detected by the LIGO-Virgo collaboration and that an electromagnetic counterpart to a gravitational wave event has been observed. GRB170817A was detected by the SPI-ACS on-board INTEGRAL and the Fermi/GBM instruments $\sim 1.7 \mathrm{~s}$ after the GW event. Following the prompt emission, INTEGRAL performed pointed observations for 5.4 days. During this time the instruments provided stringent upper limits on any electromagnetic signal in the 3 $\mathrm{keV}$ to $8 \mathrm{MeV}$ range. Interestingly, the GRB was found to be extremely subluminous.

In light of these results from GRB170817A, we have begun analysis of soft gamma-ray data $(200 \mathrm{keV}-2.6 \mathrm{MeV})$ from INTEGRAL/PICsIT. With this wide field-of-view instrument, we have begun searching for untriggered SGRBs reported by Fermi/GBM as well as preparing for real-time analysis during future LIGO-Virgo observing runs.

GRAvitational-waves Science\&technology Symposium - GRASS2018

1-2 March 2018

Palazzo Moroni, Padova (Italy)

* Speaker. 


\section{Introduction}

The INTernational Gamma-Ray Astrophysics Laboratory (INTEGRAL) is an ESA Horizon 2000 mission [1], has been providing outstanding scientific data to the whole international scientific community since its launch in 2002. The suite of on-board instruments span energies from $3 \mathrm{keV}-10 \mathrm{MeV}$ with an unprecedented sensitivity and wide fields of view (FoV) ranging from $\sim 100-1000 \mathrm{deg}^{2}$. The instruments have high time resolution $(<120 \mu \mathrm{s})$ as well as arcminute angular resolution and keV energy resolution. The large eccentricity of the satellite orbit permits long uninterrupted observations of $\sim 2.7$ days with a live-time efficiency of $>85 \%$. These features make INTEGRAL a great observatory for detecting prompt emission to electromagnetic (EM) counterparts from gravitational wave (GW) triggers from the LIGO-Virgo collaboration and also for performing subsequent follow-up observations.

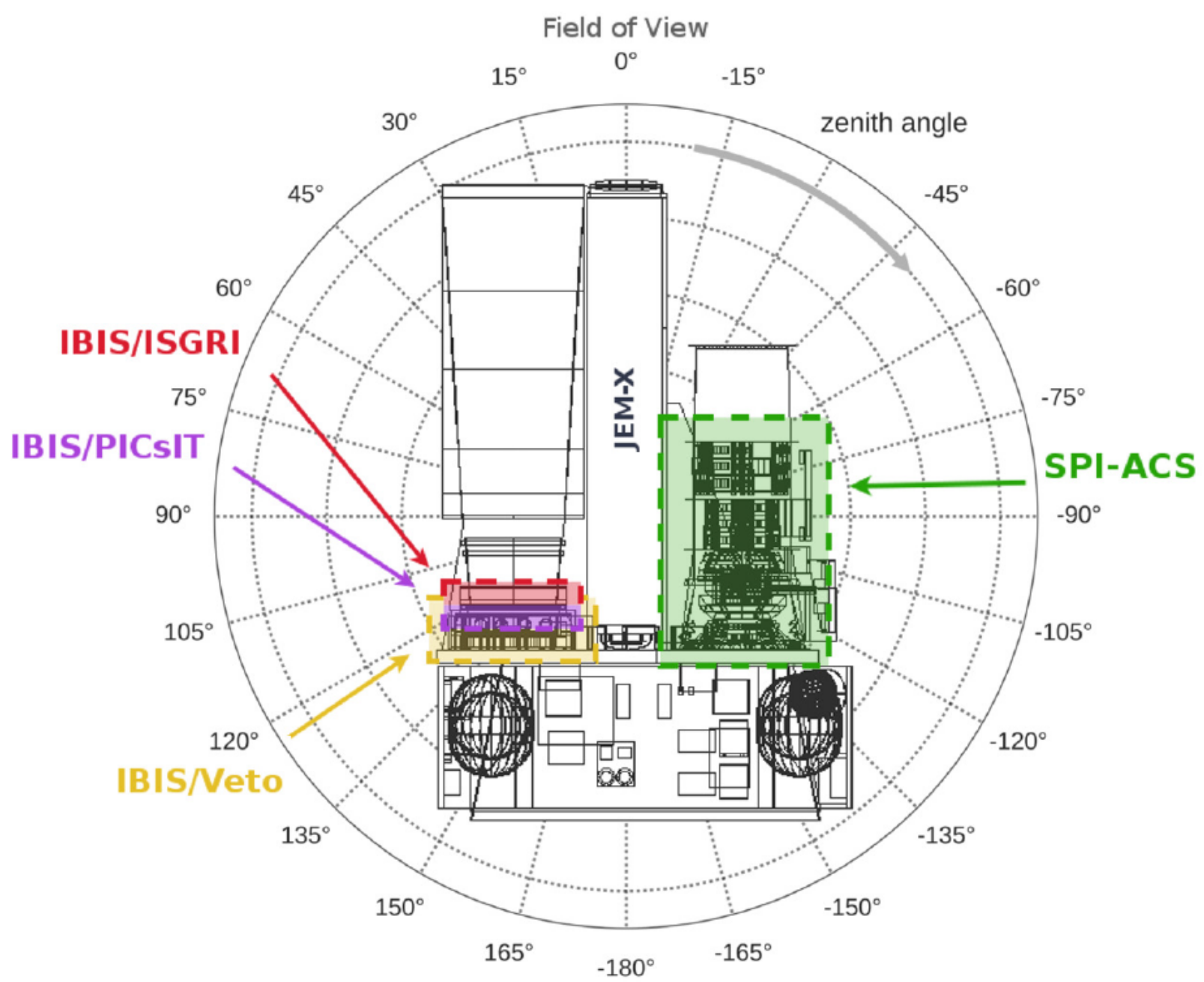

Figure 1: Diagram of INTEGRAL instruments on board the satelliate[7] 


\section{INTEGRAL All-sky Monitors}

The Anti-Coincident Shield (ACS) of the spectrometer on INTEGRAL (SPI) [2] and the PIxellated Caesium Iodide Telescope (PICsIT) operate as all-sky instruments to monitor for gamma-ray bursts (GRBs) and other impulsive events, though they were not designed for that purpose [1]. The SPI-ACS is composed of 91 Bismuth Germanate (BGO) detectors arranged to have an almost complete view of the sky [3]. The SPI-ACS has an effective area of $0.7 \mathrm{~m}^{2}$ above $\sim 75 \mathrm{keV}$ with a single energy channel and a time resolution of $50 \mathrm{~ms}$. Figure 1 shows a diagram of the INTEGRAL spacecraft with the SPI-ACS denoted in green on the right side of the figure.

The other instrument effectively working as an all-sky monitor is PICsIT, which has an area of $\sim 0.3 \mathrm{~m}^{2}$ and can detect sources out to $\sim 70^{\circ}$ off-axis [4]. PICsIT in its standard (spectral-timing) mode has a time resolution of $16 \mathrm{~ms}$ and spans energies from $\sim 200 \mathrm{keV}$ to $2.6 \mathrm{MeV}$ in 8 broad energy channels that allows for the possibility for performing rough spectral analysis. Software is being developed to perform this analysis. Also, PICsIT can observe in photon-by-photon mode to provide better temporal and spectral resolution. In Figure 1, PICsIT is located on the right side of the diagram in purple.

Because SPI-ACS and PICsIT (in standard mode) sum the signals of all the individual detectors, they can provide only rough position localization for bursts. Since the end of 2002, SPI-ACS has participated in the InterPlanetary Network (IPN) ${ }^{1}$ that employs relative differences in arrival time between multiple satellites to localize GRBs [3]. A similar localization method can be employed in helping reduce the error region from the GW signal alone, which then aides follow-up observations for instruments at lower energies that often have significantly smaller FoVs.

\section{GW170817/GRB170817A Hard X-ray Observations}

Despite no detections of prompt or after-glow emission from INTEGRAL BBH mergers observed $[5,4,6,7,8]$ (as expected), the binary neutron star (BNS) merger on 2017 August 17 at 12:40:04 UTC (GW170817) marked the first detection of an EM counterpart to a GW trigger from LIGO-Virgo collaboration. Approximately $\sim 1.7 \mathrm{~s}$ after the BNS merger, GRB 170817A was detected by INTEGRAL/SPI-ACS and Fermi/GBM at hard X-ray energies.

Temporal and spectral analyses of the GBM data found GRB 170817A to be a typical short GRB when compared to other SGRBs detected by GBM with a $\mathrm{T}_{90} \sim 2 \mathrm{~s}$ [9]. Because this event has a known distance ( $40 \mathrm{Mpc}$ [10]), unlike most SGRBs seen by GBM [9], the energetics of the burst could be calculated. Interestingly, the isotropic energy was found to be $2-6$ orders of magnitude less energetic than other SGRBs despite being about 2 order of magnitude closer.

The range of intrinsic energetics for SGRBs should have a fairly limited range since the progenitor systems are likely two neutron stars $[11,12,13,14]$. Thus seeing a large range of observed energetics is probably due to viewing the jet off-axis. In the case of GRB 170817A, both the GW signal and the hard X-ray signal estimate an observing angle of $\sim 30^{\circ}$ [9]. Such a large viewing angle disfavors the standard uniform "top-hat" model, as opposed to more complex geometries (i.e. a structured jet or a uniform jet with a cocoon), thus giving some insight into GRB jet structure, though some work is being done to interpret the hard X-ray data without the presence of a jet [15].

\footnotetext{
${ }^{1}$ http://www.ssl.berkeley.edu/ipn3/
} 

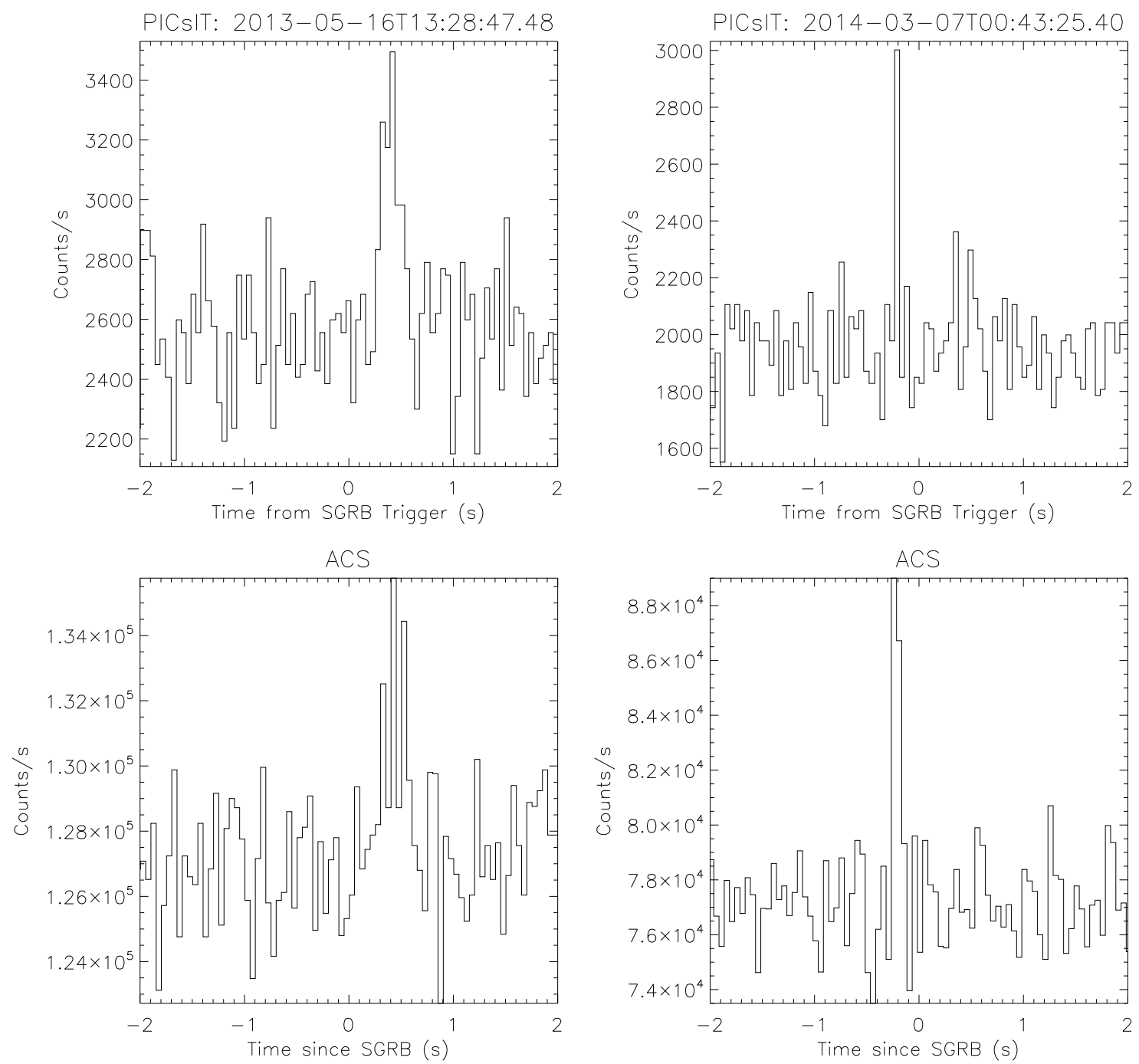

Figure 2: Un-triggered SGRBs reported by GBM that also were detected by INTEGRAL/PICsIT and SPIACS. The left column shows the $200 \mathrm{keV}-1.2 \mathrm{MeV}$ PICsIT light curve (top) and the ACS $75 \mathrm{keV}-2 \mathrm{MeV}$ light curve (bottom) of a reported SGRB with a trigger time of 2013-05-16 at 13:28:47.48 UTC. The right column shows the PICsIT and ACS light curves in the same energy ranges for a SGRB at 2014-03-07 at UTC 00:43:25.40.

Additionally, GBM estimates find that the burst could have been less luminous by $\sim 30 \%$, consistent with a distance of approximately $50 \mathrm{Mpc}$, and still have triggered on-board [9]. Consequently, there may exist a population of observed SGRBs that are too faint to trigger on-board the instrument, and thus were missed. 


\section{Current and Future Work}

Our current work has begun using past data to prepare for future observations, especially during the next LIGO-Virgo observing run. Firstly, we are searching for past INTEGRAL/PICsIT GRBs that have been previously reported by other instruments with a focus on SGRBs. An understanding of past performance of known events allows us to then make predictions about the probability of detecting EM counterparts to GW triggers.

This initial search algorithm will then be the main component for performing real-time analysis of PICsIT data, which will search for GRBs (and other transient events) as part of a larger INTEGRAL Gravitational Wave teams's work to detect prompt (and follow-up) emission related to gravitational wave events. We are also working to possibly make such PICsIT triggers immediately available online.

Additionally, the low luminosity and flux of GRB 170817A has suggested the possible existence of a population of SGRBs that are below instrument thresholds that are missed due to no on-board trigger. Initial GBM results report detecting $\sim 80$ SGRBs per year, compared to $\sim 40$ triggered events per year. Consequently, we have begun searching for sub-threshold SGRBS in PICsIT and SPI-ACS data for untriggered events reported by GBM. Figure 2 shows examples of two untriggered SGRBs with the $\mathrm{T}_{0}$ reference time from GBM. The left column is a burst on 201305-16 at 13:28:47.48 UTC. The top panel is the PICsIT data spanning $200 \mathrm{keV}-1.2 \mathrm{MeV}$, and the bottom panel is the SPI-ACS data above $\sim 75 \mathrm{keV}$. The right panel corresponds to a burst on 2014-03-07 at 00:43:25.40 UTC [16].

We have future plans for coordinated searches among the three instruments. A common search for past sub-threshold events can be used by the LIGO-Virgo collaboration to search for lowsignificance GW signals. During LIGO-Virgo observing runs, near real-time sub-threshold detections for the common search can be used to look for faint GW counterparts and lead to follow-up observations across the EM spectrum.

\section{Acknowledgements}

This work is based on observations with INTEGRAL, an ESA project with instruments and science data center funded by ESA member states (especially the PI countries: Denmark, France, Germany, Italy, Switzerland, Spain), and with the participation of Russia and the USA. The INTEGRAL SPI project has been completed under the responsibility and leadership of CNES. The SPI-ACS detector system has been provided by MPE Garching/Germany. The SPI team is grateful to ASI, CEA, CNES, DLR, ESA, INTA, NASA, and OSTC for their support. The Italian INTEGRAL team acknowledges the support of ASI/INAF agreement No. 2013-025-R.1. R.D. and A.v.K. acknowledge the German INTEGRAL support through DLR grant 50 OG 1101. A.L. and R.S. acknowledge the support from the Russian Science Foundation (grant 14-22- 00271). A.D. is funded by Spanish MINECO/FEDER grant ESP2015-65712-C5-1-R. We acknowledge the continuous support by the INTEGRAL Users Group and the exceptionally efficient support by the teams at ESAC and ESOC for the scheduling of the targeted follow-up observations. We are grateful to the LVC and Fermi-GBM teams for their suggestions on earlier versions of this Letter. The research 
leading to these results has received funding from the European Union's Horizon 2020 Programme under the AHEAD project (grant agreement n. 654215).

\section{References}

[1] C. Winkler, The INTEGRAL mission

[2] G. Vedrenne, et al., SPI: The Spectrometer aboard INTEGRAL,A\&A, 411, L63 (2003)

[3] A. von Kienlin, et al., NTEGRAL Spectrometer SPI's GRB detection capabilities. GRBs detected inside SPI's FoV and with the anticoincidence system ACS, A\&A, 411, 299 (2003)

[4] V. Savchenko, et al., INTEGRAL IBIS, SPI, and JEM-X observations of LVT151012, A\&A, 603, 46 (2017)

[5] V. Savchenko, et al., INTEGRAL Upper Limits on Gamma-Ray Emission Associated with the Gravitational Wave Event GW150914, ApJ, 820, 36 (2016)

[6] V. Savchenko, et al., INTEGRAL Observations of GW170104, ApJ, 846, 23 (2017)

[7] V. Savchenko, et al., LIGO/Virgo G297595: INTEGRAL search for a prompt gamma-ray counterpart, GCN Cir, 21478 (2017)

[8] V. Savchenko, et al., INTEGRAL Detection of the First Prompt Gamma-Ray Signal Coincident with the Gravitational-wave Event GW170817, ApJ, 848, 15 (2017)

[9] A. Goldstein, et al., An Ordinary Short Gamma-Ray Burst with Extraordinary Implications: Fermi-GBM Detection of GRB 170817A, ApJ, 848, 14 (2017)

[10] LIGO Scientific Collaboration \& Virgo Collaboration et al., GCN, 21513 (2017)

[11] S. I. Blinnikov, et al., Exploding Neutron Stars in Close Binaries, SvAL, 10, 177 (1984)

[12] E. Nakar, Short-hard gamma-ray bursts, PhR, 442, 166 (2007)

[13] N. Gehrels \& P. Meszaros, Gamma-Ray Bursts, Sci, 337, 932 (2012)

[14] E. Berger, Short-Duration Gamma-Ray Bursts, ARA\&A, 52, 43 (2014)

[15] A. Neronov, et al., in preperation

[16] B. P. Abbott, et al., Gravitational Waves and Gamma-Rays from a Binary Neutron Star Merger: GW170817 and GRB 170817A Scientific Collaboration and Virgo Collaboration, Fermi Gamma-ray Burst Monitor, and INTEGRAL, ApJL, 848, L13 (2017). 